\title{
Fractional Order Modeling and Control of Twin Rotor Aero Dynamical System using Nelder Mead Optimization
}

\author{
Salman Ijaz ${ }^{\dagger}$, Mirza Tariq Hamayun*, Lin Yan** and Muhammad Faisal Mumtaz*** \begin{abstract}
input multi output twin rotor aerodynamic system. Dynamics of the considered system are highly nonlinear and there exists a significant cross-coupling between the horizontal and vertical axes (pitch \& yaw). In this paper, a fractional order model of twin rotor aerodynamic system is identified using input output data from nonlinear system. Based upon identified fractional order model, a fractional order PID controller is designed to control the angular position of level bar of twin rotor aerodynamic system. The parameters of controller are tuned using Nelder-Mead optimization and compared with improvement in the performance of fractional order PID controller as compared to a classical PID controller.
\end{abstract} \\ Abstract - This paper presents an application of fractional order controller for the control of multi \\ particle swarm optimization techniques. Simulation results on the nonlinear model show a significant
}

Keywords: Fractional order controller, Twin rotor, Nelder-Mead optimization, Classical PID

\section{Introduction}

Twin rotor aerodynamic system (TRAS) has gained a significant attention of the researchers in the past few decades. TRAS is highly nonlinear and there exist a strong cross-coupling between the horizontal and vertical axis of rotors. So it is always been a challenging task for the researcher to achieve a precise level of accuracy in the control of such systems. TRAS is a multi-input multioutput (MIMO) laboratory setup (Fig. 1) which resembles in functional behavior to the helicopter.

In this study, a fractional order PID (FOPID) controller is designed to control pitch and yaw position of nonlinear TRAS model using Nelder-Meads (N-M) optimization technique. For such type of MIMO system where the crosscoupling between the axes has strongly influenced on system dynamics and robust stability is the prior requirement of application, classical controllers are often failed to achieve desired performance. The choice of fractional order controller (FOC) looks promising due to its better transient response result compared to integral order controller.

Moreover, it provides less overshoot and is less sensitive to variation of the system parameters when FOC applied to fractional order systems (FOS) [1].

In the recent years, a substantial studies and applications are being focused on FOPID controller. A simple structure

$\dagger$ Corresponding Author: School of Automation and Electrical Engineering, Beihang University, China.(salman_ijaz830@hotmail.com)

* Dept. of Electrical Engineering, COMSATTS Lahore, Pakistan. (mhamayun@ciitlahore.edu.pk)

** School of Automation and Electrical Engineering, Beihang University, China.(linyan@buaa.edu.cn)

*** Dept. of Electrical Engineering, ISP Multan Pakistan. (faisalmumtaz2014@yahoo.com)

Received: October 4, 2015; Accepted: April 18, 2016 controller is found very effective as compared to other classical controllers [2]. FOPID controller has found its application in control of single input single output (SISO) systems such as speed control of DC motor in [3-4], torsional system's backlash vibration suppression control in [5], bioreactor control system in [6] and position tracking control of electro hydraulic servo system (EHSS) in [7]. FOPID controller has also been applied to MIMO system in [8-9]. All such applications were required optimally tuned controller parameters based upon the optimization technique, search space dimension and fitness functions.

PID controller due to its ease in design and implementation has been applied to nonlinear TRAS [10-13] and its performance was greatly improved by optimal tuning controller parameters using genetic algorithm (GA) optimization technique. An optimal state feedback controller approach was considered in [14] to control azimuth and elevation angle and height of TRAS when moving upward, whereas output feedback control strategy was used in [15] to control pitch and yaw position of nonlinear TRAS. A higher order 2-DOF- controller was designed and implemented on TRAS laboratory setup in [16]. The controller was capable to account for model uncertainties and was able to maintain stability. The difficulty in implementation of higher order controller was also addressed.

In this paper, a FOPID controller is designed to control the pitch and yaw angles of TRAS while minimizing the effect of cross-coupling between the two axes and robustness against external disturbances in the control channel. A nonlinear mathematical model of TRAS is first linearized in both integer order and fractional order model representation. A novel contribution in this paper is fractional order model identification based on input output 
data from nonlinear TRAS. Based upon that model, FOC is designed separately for pitch and yaw. The parameters of controller are tuned using N-M optimization and particle swarm optimization (PSO) technique. A performance index is formulated to evaluate the controller performance. To validate the effectiveness of the designed controller, it is tested on the nonlinear model of TRAS. Simulation results reveal remarkable improvement in performance using FOPID controller designed using N-M technique compared to classical PID controller and other FOPID design technique included in the literature. Moreover it has been analyzed that FOS gives better representation of system dynamics as compared to integral order.

The rest of the paper is organized as follow: Problem formulation and nonlinear dynamical model of TRAS is presented in Section 2. A detailed description of control strategy is illustrated in section 3 that includes fractional order model identification and FOC design procedure for nonlinear TRAS. Section 4 contains a brief description of optimization technique applied to tune controller parameters. FOPID controller design and simulation results are presented in section 5. Finally the concluding remarks are given in section 6 .

\section{Problem Formulation and Dynamical Model of TRAS}

The mechanical structure of an actual TRAS model [10] is shown in Fig. 1, where the two rotors are connected to the ends of the beam. One is considered as main rotor and other as tail rotor. The beam can move freely in horizontal and vertical planes. The equilibrium point of the beam is decided by a counterbalance and one end of its arm is connected to the beam at pivot point and other end with a weight. Two DC motors are used to drive main and tail rotors. The main rotor provides the vertical movement and rotates about the pitch axis whereas the tail rotor turns the beam right or left about the yaw axis. A nylon screw is mounted near the pivot point that restricts either the horizontal or the vertical degree of freedom to 1-DOF.The whole unit is placed on tower that allows safe helicopter

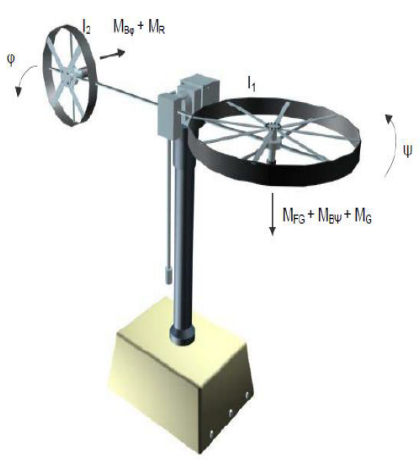

Fig. 1. MIMO Twin rotor aerodynamics system
Table 1: Model parameters and values of TRAS [14]

\begin{tabular}{l|c}
\hline \multicolumn{1}{c}{ Parameters } & Values \\
\hline $\mathrm{I}_{1}$ Moment of inertia of vertical rotor & $6.8 e^{-2} \mathrm{~kg} \cdot \mathrm{m}^{2}$ \\
\hline $\mathrm{I}_{2}$ Moment of inertia of horizontal rotor & $2 e^{2} \mathrm{~kg} \cdot \mathrm{m}^{2}$ \\
\hline$a_{1}$ Static characteristic parameter & 0.0135 \\
\hline$b_{1}$ Static characteristic parameter & 0.0924 \\
\hline$a_{2}$ Static characteristic parameter & 0.02 \\
\hline$b_{2}$ Static characteristic parameter & 0.09 \\
\hline$M_{g}$ Gravity momentum & $0.32 \mathrm{Nm}$ \\
\hline$B_{1 \psi}$ Friction momentum function parameter & $6 e^{-3} \mathrm{~N} \cdot \mathrm{m} \cdot \mathrm{s} / \mathrm{rad}$ \\
\hline$B_{2 \psi}$ Friction momentum function parameter & $1 e^{-3} \mathrm{~N} . \mathrm{m} \cdot \mathrm{s} / \mathrm{rad}$ \\
\hline$B_{1 \phi}$ Friction momentum function parameter & $1 e^{-1} \mathrm{~N} \cdot \mathrm{m} \cdot \mathrm{s} / \mathrm{rad}$ \\
\hline$B_{2 \phi}$ Friction momentum function parameter & $1 e^{-2} \mathrm{~N} \cdot \mathrm{m} \cdot \mathrm{s} / \mathrm{rad}$ \\
\hline$K_{g y}$ Gyroscope momentum parameter & $0.05 \mathrm{~s} / \mathrm{rad}$ \\
\hline$k_{1}$ gain of motor 1 & 1.1 \\
\hline$k_{2}$ gain of motor 2 & 0.8 \\
\hline$T_{11}$ motor 1 denominator parameter & 1.1 \\
\hline$T_{10}$ motor 1 denominator parameter & 1 \\
\hline$T_{21}$ motor 2 denominator parameter & 1 \\
\hline$T_{20}$ motor 2 denominator parameter & 1 \\
\hline$k_{c}$ Cross reaction momentum gain & -0.2 \\
\hline$T_{o}$ Cross reaction momentum parameter & 3.5 \\
\hline$T_{p}$ Cross reaction momentum parameter & 2 \\
\hline
\end{tabular}

control experiments.

Using the Fig. 1, the nonlinear dynamical equations have been written and parameter values of TRAS are shown in Table 1. In [14], the momentum equations for the vertical movement are derived as

$$
\ddot{\psi}=f(\psi, \phi, \tau)=\frac{1}{I_{1}}\left[M_{1}-M_{F G}-M_{B \psi}-M_{G}\right]
$$

Where $M_{1}$ is the nonlinear characteristic induced by the rotor and is approximated by a $2^{\text {nd }}$ order polynomial given by

$$
M_{1}=a_{1} \tau_{1}^{2}+b_{1} \tau_{1}
$$

where $a_{1}$ and $b_{1}$ are static characteristic parameters and the expression for the $\tau_{1}$ term is explained in the sequel.

The weight of helicopter generates gravity torque about pivot point and can be described as

$$
M_{F G}=M_{g} \sin \psi
$$

where $M_{g}$ is the gravity momentum. The frictional torque $M_{B_{\psi}}$ can be estimated as

$$
M_{B \psi}=B_{1 \psi} \dot{\psi}-\frac{0.0326}{2} \sin 2 \psi \dot{\phi}^{2}
$$

where $B_{1 \psi}$ is friction momentum parameter.

As Coriol is force cause the occurrence of gyroscopic torque $M_{G}$ and is dominated when moving main rotor turns its position in azimuth direction and can be described as

$$
M_{G}=K_{g y} M_{1} \dot{\phi} \cos \psi
$$


where $K_{g y}$ is termed as gyroscopic momentum paramter. Note that (5) shows the cross-coupling effect in the horizontal plane.

The DC motor 1 and the electrical control circuit can be approximated by a $1^{\text {st }}$ order transfer function and the motor momentum in Laplace domain is described as

$$
\tau_{1}=\frac{k_{1}}{T_{11} s+T_{10}} u_{1}
$$

where $k_{1}$ is the gain of motor 1 and $T_{11}, T_{10}$ are motor 1 denominator parameters. $u_{1}$ is the motor input voltage.

In a similar fashion to Eq. (1)-(5), the net torque produced in horizental plane motion is described by a dynamical equations as

$$
\ddot{\phi}=f(\phi, \psi, \tau)=\frac{1}{I_{2}}\left[M_{2}-M_{B \phi}-M_{R}\right]
$$

where $M_{2}$ is the nonlinear stationary characteristic similar to main rotor and is approximated by

$$
M_{2}=a_{2} \tau_{2}^{2}+b_{2} \tau_{2}
$$

where $a_{2}$ and $b_{2}$ are the statistic characteristic parameters.

Similar to Eq. (4), friction torque is calculated as

$$
M_{B \phi}=B_{2 \phi} \dot{\phi}
$$

where $\mathrm{B}_{2 \phi}$ is friction momentum function parameter. The cross reaction momentum $\mathrm{M}_{\mathrm{R}}$ is approximated by

$$
\mathrm{M}_{\mathrm{R}}=\frac{\mathrm{k}_{\mathrm{c}}\left(\mathrm{T}_{\mathrm{o}} \mathrm{s}+1\right)}{\mathrm{T}_{\mathrm{p}} \mathrm{s}+1} \tau_{1}
$$

where $\mathrm{k}_{\mathrm{c}}$ is cross reaction momentum gain. $\mathrm{T}_{\mathrm{o}}$ and $\mathrm{T}_{\mathrm{p}}$ are cross reaction momentum parameters. The DC motor 2 and the electrical circuit transfer function with electric circuit transfer function is given in Laplace domain in [14] as

$$
\tau_{2}=\frac{\mathrm{k}_{2}}{\mathrm{~T}_{21} \mathrm{~s}+\mathrm{T}_{20}} \mathrm{u}_{2}
$$

where $\mathrm{k}_{2}$ is gain of motor 2. $\mathrm{T}_{21}$ and $\mathrm{T}_{20}$ are motor denominator parameters. $\mathrm{u}_{2}$ is the voltage applied to motor 2.

The state vector of TRAS is $\mathrm{x}=\left[\psi \dot{\psi} \phi \dot{\phi} \tau_{1} \tau_{2} \mathrm{M}_{\mathrm{G}}\right]^{\mathrm{T}}$, where $\psi$ is pitch angle, $\dot{\psi}$ is pitch rate, $\phi$ is yaw angle, $\dot{\phi}$ is yaw rate, $\tau_{1}$ is momentum of main rotor, $\tau_{2}$ is momentum of tail rotor and $\mathrm{M}_{\mathrm{G}}$ is gyroscopic torque. The input control vector is $\mathrm{u}=\left[\mathrm{u}_{\mathrm{v}} \mathrm{u}_{\mathrm{h}}\right]^{\mathrm{T}}$, where $\mathrm{u}_{\mathrm{v}}$ and $\mathrm{u}_{\mathrm{h}}$ are the input voltages of main rotor and tail rotor respectively. The controlled output vector is $\mathrm{y}=[\psi \phi]^{\mathrm{T}}$.

The nonlinear mathematical model of TRAS described from (1-11) represents the system dynamics. Then nonlinear model is first approximated in fractional order LTI model representation prior to the FOC design. Later on, the designed controller is implemented on nonlinear dynamical model to validate performance.

\section{Control Strategy}

The TRAS is characterized by highly nonlinear, complex and inaccessibility of some states for measurements and hence can be thought of as a challenging problem due to significant cross coupling between the horizontal and vertical axes. Keeping in view the model complexity and to improve the performance of classical PID controller, a selection of FOPID controller is promising. The proposed controller is expected to improve the performance of system in terms of stability margin, operating frequency range and disturbance rejection capability. Several performance criteria are available to evaluate the controller performance. This research includes integrated absolute error (IAE) to evaluate the system performance because it tends to produce the response with less sinusoidal oscillation. The transient analysis of controller is based upon specifications such as settling time $\left(\mathrm{T}_{\mathrm{s}}\right)$, rise time $\left(\mathrm{T}_{\mathrm{r}}\right)$, overshoot $\left(\sigma_{\mathrm{e}}\right)$, steady state error $\left(\mathrm{E}_{\mathrm{SS}}\right)$ and integrated absolute error (IAE). The tools used for frequency domain analysis are gain margin $\left(\mathrm{G}_{\mathrm{M}}\right)$, phase margin $\left(\mathrm{P}_{\mathrm{M}}\right)$ and operating bandwidth. The performance criteria $K(s)$ is defined by assigning weight to each factor as

$$
\begin{aligned}
K(s)= & w_{1} \sigma_{e}+w_{2} E_{s s}+w_{3} T_{s}+w_{4} T_{r}+w_{5} \int|e| d_{t} \\
& +\frac{w_{6}}{G_{M}}+\frac{w_{7}}{P M}
\end{aligned}
$$

In coming section, fractional order model identification method of TRAS and brief description of FOC are discussed.

\subsection{Fractional order model identification of TRAS}

Fractional calculus (FC) is the part of mathematics that outspreads derivation and integration to a random order. It simplifies the traditional differential operator $D t^{\alpha} \equiv$ $d^{\alpha} / d t^{\alpha}$ to a fractional operator, where $\alpha$ is a real number. However, its characteristic complication reduces the use of the associated ideas [17].

In order to design and analyze appropriate controller, it is very important to know about accurate mathematical model of a system. Due to nonlinear system dynamics, the accurate mathematical model is higher order. The Laplace transformation approach gives a closed approximation of higher order system but it is not accurate as fractional order representation of system [18]. It is proved in [19] that any dynamical system whether it is lumped or continuous can be represented in fractional order. A fractional order model representation of system is more adequate representation than integral order model. Some methods of fractional order model identification in time domain are discussed in [20]-[26] and in frequency domain in [27, 28].

Overall, FOS can be expressed as LTI fractional order differential (FOD) equation of the form: 


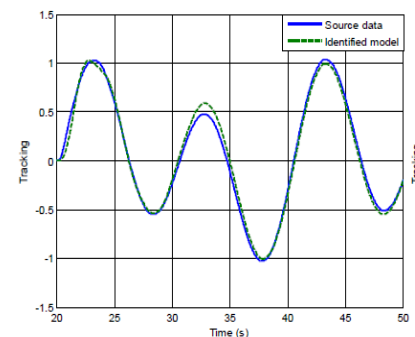

(a)

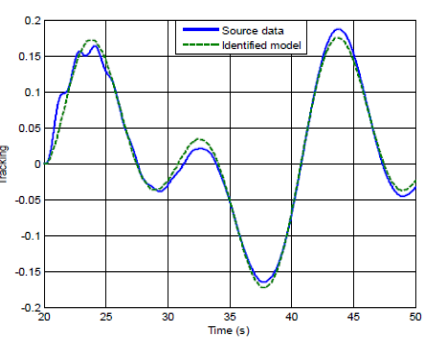

(b)
Fig. 2. Fractional order model identification Curve for (a) pitch and (b) yaw

$$
\begin{aligned}
& a_{n} D_{t}^{\beta_{n}} y(t)+a_{n-1} D_{t}^{\beta_{n-1}} y(t) \ldots a_{0} D_{t}^{\beta_{0}} \ldots d_{m} D_{t}^{\alpha_{m}} u(t) \\
& \quad+d_{m-1} D_{t}^{\alpha_{m-1}} u(t)+\cdots+d_{0} D_{t}^{\alpha_{0}} u(t)
\end{aligned}
$$

Or by a continuous transfer function of the form

$$
G(s)=\frac{d_{m} s^{\alpha_{m}}+d_{m-1} s^{\alpha_{m-1}+\cdots . .+d_{0} s^{\alpha_{0}}}}{a_{n} s^{\beta_{n}+a_{n-1} s^{\beta}-1+\cdots . .+a_{0} s^{\beta_{0}}}}
$$

where $\alpha_{k}, \beta_{k}$ are real numbers and $a_{k}, d_{k}$ are random constants.

This section presents the fractional order model identification of TRAS. Two approaches are being followed for system identification: one way is to linearize nonlinear term of given model using any linearization technique. The other is indirect approach using input-output data from nonlinear real time system.

In this paper, the fractional order model of TRAS for pitch and yaw is identified in closed loop configuration of system using indirect approach. Input-output data set is collected from nonlinear TRAS system in closed loop configuration. The data is then fed to fractional order identification FOMCON toolbox in MATLAB [25]. Grünwald-Letnikov method of approximation is used and can be defined as

$$
D_{t}^{\alpha} f(t)=\lim _{\mathrm{h} \rightarrow 0} \frac{1}{\mathrm{~h}^{\alpha}} \sum_{\mathrm{i}=0}^{\mathrm{k}}(-1)^{\mathrm{j}}\left(\begin{array}{l}
\alpha \\
\mathrm{j}
\end{array}\right) \mathrm{f}(\mathrm{t}-\mathrm{jh})
$$

The open loop fractional order model is extracted later on from the identified closed loop model. The model identification curve is shown in Fig. 2.

The fractional order transfer function representation of system is given by

$$
\begin{aligned}
G_{11} & =\frac{\psi}{u_{v}}=\frac{1}{1.4 s^{2.5}-7.1 s^{1.3}+29 s-20.1 s^{0.9}+4.3 s^{0.2}+0.5} \\
G_{22} & =\frac{\phi}{u_{h}}=\frac{1}{1.5 s^{2.5}-1.2 s^{1.4}+17.3 s^{1.1}-15.9 s^{0.7}+6.8 s^{0.3}-0.4 s^{0.2}}
\end{aligned}
$$

and cross-coupling between the two axes is modeled as

$$
\begin{aligned}
& G_{12}=\frac{\phi}{u_{v}}=\frac{1}{1.9 s^{2.3}-4.9 s^{0.9}+4.6 s^{0.8}+7.9 s^{0.3}-0.2 s^{0.1}} \\
& G_{21}=\frac{\psi}{u_{h}}=\frac{1}{-8.8 s+38.7 s^{0.7}+159.5 s^{0.1}+0.4}
\end{aligned}
$$

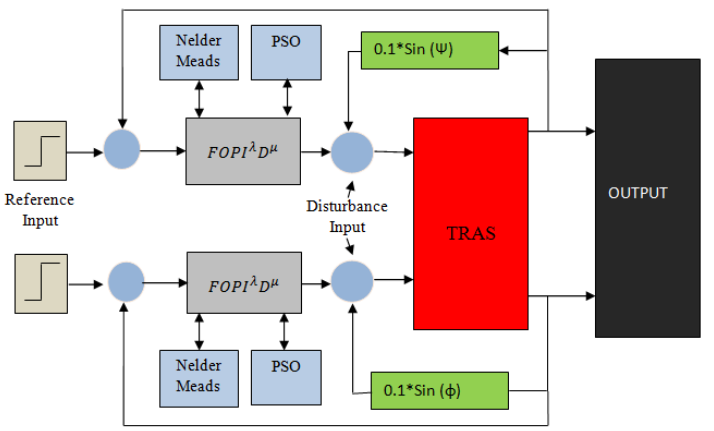

Fig. 3. FOPID control strategy for TRAS

The fractional order model in (16-17) is used as benchmark in the design of FOPID controller.

\subsection{Fractional order PID controller}

FOC was first introduced by Oustaloup (1991) and developed as crone controller. The transfer functien representation of FOPID controller is

$$
G_{c}(s)=K_{P}+K_{i} s^{-\lambda}+K_{d} s^{\mu}, \mu, \lambda>0
$$

From (18), it is clear that FOPID controller has five tuning parameters that are Proportional gain $\left(K_{p}\right)$, Integral gain $\left(K_{i}\right)$, Derivative gain $\left(K_{d}\right)$, Integral fractional power $(\lambda)$ and derivative fractional power $(\mu)$. Clearly, depending on the values of $\lambda$ and $\mu$, various number of choices for controller development are available (defined continuously on the $(\mu, \lambda)$-plane).

There is an increasing trend of researchers on FOPID controller design for various applications. The proposed controller has simple structure. Due to the availability of additional adjustable parameters (i.e. $\lambda, \mu$ ), the FOPID controller has been found effective as compared to classical PID controller. A block diagram representation of the control strategy is shown in Fig. 3. It provides robustness under the variation of system parameters, controller parameters and external load [29-30]. So it is quite suitable for TRAS as there are some uncertain parameters that classical PID was found less effective to overcome.

In the application of FOPID controller, mainly two type of problem arises. The first one is the model approximation or realization and the other is to tune the controller parameters. Two techniques are used extensively for model approximation. The first one is interpolation technique and other is curve fitting technique such as Oustaloup filter [31]. The problem of parameter tuning is more critical than the first one. So there is a need of some optimization technique that can tune the system parameters according to desire specification.

In this paper, the FOPID controller is applied to control the angular position of level bar of TRAS. The problem of parameters optimization is solved using Nelder-Mead optimization criteria. Fitness function is calculated according 
to criteria given in (12). FOPID controller is applied using FOMCON toolbox in MATLAB [32]. The toolbox for MATLAB is intended to help developing fractional order model and controllers and to assess their performance. It allows simulating, optimizing and analyzing FOPID controllers easily via its functions

\section{Parameter Tuning using Optimization Technique}

The section discusses the N-M algorithm and PSO technique to tune the parameters of FOPID controller. The objective function is to minimize the performance index defined in (12) such that closed loop system is internally stable. The comparison of both techniques is done in simulation results.

\subsection{Nelder mead optimization technique}

In this section, the parameters of FOPID controller are tuned using N-M optimization [33]. It is a simplex based algorithm to find local minima and was first proposed by Nelder and Mead in 1965.This method is computationally simple so that it is recognized as well known in the field of parameter optimization. This method has the closest resemblance to differential evolution (DE) and PSO [34]. This method applies pattern search approach of $n+1$ dimensional shape and does not require derivative information to minimize the function of $n$ variables. The search done by N-M is based on geometric operation (reflection, expansion, contraction, and shrinking) on the corner sets of point seen as corner of n-dimensional polygon to determine which point in space to evaluate next. The overall behavior of N-M algorithm is to expand or focuses the search adaptively on the basis of topology of fitness landscape.

As the original versions of DE and PSO, N-M algorithm requires the search space to be continuous and the points in space to be represented as vectors of real numbers [35]. There is no generalization of N-M algorithm to combinational space.

Table 2. Nelder-mead algorithm to tune fopid parameter

\begin{tabular}{c|c}
\hline IF $\mathrm{f}(\mathrm{R})<\mathrm{f}(\mathrm{G})$, The case(i) & either reflect or extend $\}$ \\
ElSE perform Case(ii) & either contract or shrink \\
\hline BEGIN $\{$ Case $(\mathrm{i})\}$ & BEGIN $\{$ Case $(\mathrm{ii})\}$ \\
IF $\mathrm{f}(\mathrm{B})<\mathrm{f}(\mathrm{R}) \mathrm{THEN}$ & $\mathrm{IF} \mathrm{f}(\mathrm{R})<\mathrm{f}(\mathrm{W}) \mathrm{THEN}$ \\
Replace W with $\mathrm{R}$ & Replace $\mathrm{W}$ with $\mathrm{R}$ \\
ELSE & Computer $\mathrm{C}=(\mathrm{W}+\mathrm{M}) / 2$ \\
Compute E and $\mathrm{f}(\mathrm{E})$ & Or $\mathrm{C}=(\mathrm{M}+\mathrm{R}) / 2$ and $\mathrm{f}(\mathrm{C})$ \\
IF $\mathrm{f}(\mathrm{E})<\mathrm{f}(\mathrm{B})$ THEN & IF $\mathrm{f}(\mathrm{C})<\mathrm{f}(\mathrm{W}) \mathrm{THEN}$ \\
Replace W with E & Replace W with C \\
ELSE & ELSE \\
Replace W with R & Compute S and $\mathrm{f}(\mathrm{S})$ \\
END IF & Replace W with S \\
END IF & Replace G with M \\
END $\{$ Case $(\mathrm{i})\}$ & END $\{$ Case $(i i)\}$ \\
\hline
\end{tabular}

$\mathrm{N}-\mathrm{M}$ technique has been applied to tune the parameters of PID controller designed for position tracking control of EHSS [36]. Simulation results show better tacking performance as compared to self-tuning fuzzy approach. The procedure for N-M search is shown in Table 2.

In this paper, the N-M technique is applied to tune $K_{p}, K_{i}, K_{d}, \lambda$ and $\mu$ parameters of FOPID controller. The tool for parameter identification using N-M techniques is discussed in [37]. The performance criteria developed in (12) is used as a fitness function.

\subsection{Particle swarm optimization technique}

PSO is population based stochastic optimization technique inspired by social behavior of bird flocking and fish schooling [38]. It is a computational based method that optimizes the problem by improving the candidate function iteratively. In PSO, each individual is called a particle and each particle updates its position according to its own flight experience and the companion experience. Each particle is treated as a point in n-dimensional subspace, where the $i_{t h}$ particle is represented as $X_{I}=$ $\left[x_{i_{1}}, x_{i_{2}}, \ldots \ldots x_{i_{n}}\right]$. The previous best position is called $p_{\text {best }}$ and is represented as $P_{I}=\left[p_{i_{1}} \ldots \ldots p_{i_{n}}\right]$. The best particle amongst all in the population is called $g_{\text {best }}$. The velocity vector is updated according to current position of each particle. The particles are updated according to the following equations:

$$
\begin{aligned}
& v_{i_{n}}^{m+1}=w \cdot v_{i_{n}}^{m}+c_{1} \cdot \operatorname{rand}() \cdot\left(p_{i_{n}}-x_{i_{n}}\right)+c_{2} \cdot \operatorname{rand}() . \\
& \left(P_{g d}^{m}-x_{i_{n}}^{m}\right) x_{i_{n}}^{m+1}=x_{i_{n}}^{m}+v_{i_{n}}^{m+1}
\end{aligned}
$$

where $c_{1}$ and $c_{2}$ are positive constants and $\operatorname{rand}()$ is a random function between 0 and $1 . P_{g d}$ is the global best value of particle and $w$ is the inertial weight. Equation (19) is used to find the new velocity and position update, where $m$ represents the number of iterations.

In this paper, PSO is adopted to tune the parameters of FOPID controller and is compared with N-M technique. The optimized value of parameters is shown in Table 4 .

\section{FOPID Controller Design and Nonlinear Simulation Results}

This section covers the FOPID controller design and simulation results based on the nonlinear model of TRAS.

\subsection{FOPID controller design}

This subsection explains the design strategy of pitch and yaw angles control of TRAS using FOPID and PID controller. The overall setup of TRAS is MIMO so two separate controllers are designed, one for the control of pitch angle in vertical direction and other to control yaw in 
Table 3. PID Parameters

\begin{tabular}{c|c|c}
\hline Parameters & Pitch & Yaw \\
\hline$K_{p}$ & 3.01 & 2 \\
\hline$K_{i}$ & 8.24 & 0.5 \\
\hline$K_{d}$ & 10.11 & 5 \\
\hline
\end{tabular}

Table 4. FOPID parameters

\begin{tabular}{c|c|c|c|c}
\hline Parameters & $\begin{array}{c}\text { Pitch } \\
(\mathrm{N}-\mathrm{M})\end{array}$ & $\begin{array}{c}\text { Pitch } \\
(\mathrm{PS} 0)\end{array}$ & $\begin{array}{c}\text { Yaws (N- } \\
\mathrm{M})\end{array}$ & $\begin{array}{c}\text { Yaw } \\
(\mathrm{PSO})\end{array}$ \\
\hline $\mathrm{Kp}$ & 28.4971 & 3.87 & 4.901 & 2.8 \\
\hline $\mathrm{Ki}$ & 16.012 & 5.21 & 3.491 & 0.75 \\
\hline $\mathrm{Kd}$ & 39.103 & 14.421 & 5.942 & 5.1 \\
\hline$\lambda$ & 0.62 & 0.71 & 0.75 & 1.2 \\
\hline$\mu$ & 1.103 & 0.903 & 0.91 & 0.83 \\
\hline
\end{tabular}

horizontal direction. A block diagram representation of overall controller design strategy is shown in Fig. 3. In this design, N-M optimization and PSO techniques are adopted to tune the controller parameters under the following constraints; Rise time is limited to $5 \mathrm{~s}$, settling time is set to $10 \mathrm{~s}, \%$ overshoot not more than $20 \%$ is allowed, gain margin is set to $10 \mathrm{~dB}$ and phase margin to $60^{\circ}$. The algorithm provides pattern search approach with $\mathrm{k}+1$ dimensional shape, where $\mathrm{k}$ is the number of parameters to be optimized ( $\mathrm{k}=5$ in our case). Initially, the value of parameters of FOPID controller is set to $\mathrm{K}_{\mathrm{p}}=\mathrm{K}_{\mathrm{i}}=\mathrm{K}_{\mathrm{d}}=$ $1, \lambda=\mu=0.5$. The algorithms provide online tuning of controller parameters based upon system model. In PSO tuning algorithm, the range of particles is set from 0 to 100 . The size of swarm and maximum bird step are assumed to be 50. The optimized value of PID and FOPID parameters are shown in Table 3 and Table 4. N-M optimization method selected one vertex from search area on each iteration. PSO technique easily falls in optimal solution but due to the requirement of various particles the speed of computation is slow.

By using the optimal value of parameters, the controller performance is tested in closed-loop configuration under various reference tracking signals. Simulation results are discussed in detail in the upcoming section.

\subsection{Simulation results}

Here the simulation results of FOPID controller applied to control the pitch and yaw angles of MIMO TRAS system are presented. Two optimization techniques (N-M and PSO) are used in this research and simulation results are compared in the end. To check the robustness of the FOPID controller the disturbance signals (sinusoidal functions of states $(\psi, \phi))$ are also applied at the input control input channel. The feasibility and the tracking performance of the designed controllers are checked by applying different reference signals. Simulation results are based upon nonlinear model of TRAS and are compared with conventional PID and FOPID approach applied in [9].

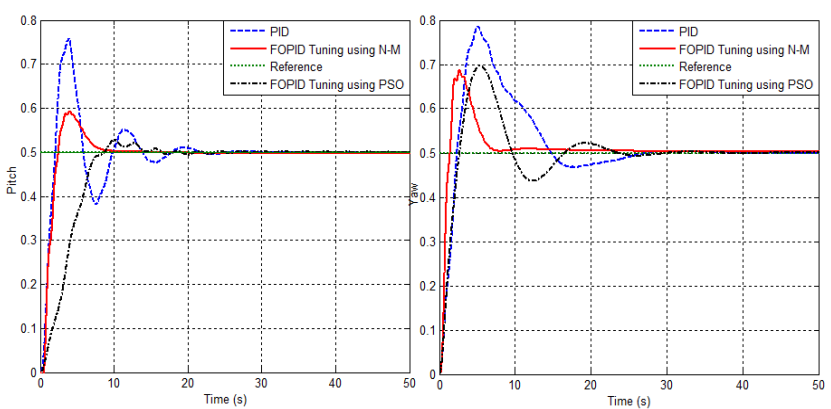

Fig. 4. Tracking Performance of nonlinear TRAS at step Input

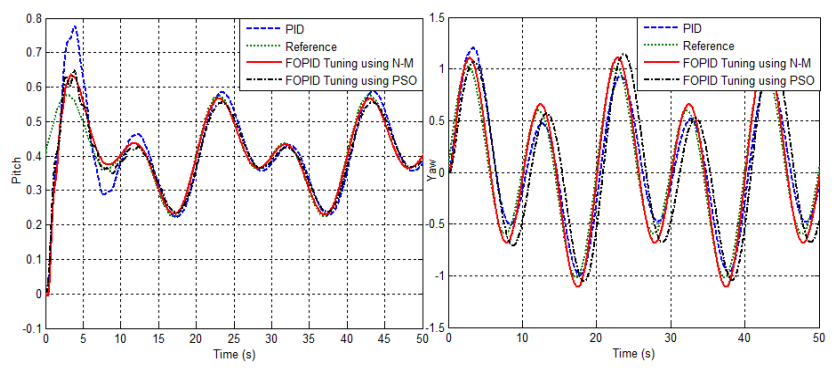

Fig. 5 Tracking performance of nonlinear TRAS at sinusoidal input

Simulation results at various reference signals show the dominant performance of proposed methodology. It may also be noted that fractional order model of TRAS gives better representation of system dynamics compared to integer order model. Also N-M optimization technique applied to nonlinear TRAS gives more optimally tuned controller parameters as compared to PSO.

In the simulation results shown in Fig. 4, step reference commands are applied to pitch up (to increase height) and at the same time movement in the yaw direction to change heading. It is clear from the results that FOPID tuned using $\mathrm{N}-\mathrm{M}$ has improved performance in terms of transient and ensures to reach steady state condition earlier as compared to FOPID tuned using PSO and conventional PID controller. Moreover, FOPID controller possesses less overshoot as compared to PID controller.

In Fig. 5, a reference trajectory for both pitch and yaw is take $r(t)=0.1 \sin (0.1 t)+0.1 \sin (0.05 t)+0.1 \sin (0.02 t)$. Simulation result shows better reference tracking of FOPID controller on time varying trajectory comparable to PID controller. In case of yaw, the tracking of PID controller is comparable to FOPID controller. But the FOPID controller required less control effort to achieve desired trajectory as compared to PID controller shown in Fig. 8.

The simulation results presented in Fig. 6 show that cross-coupling effects are minimized while applying the step reference command to pitch up only and yaw reference command is zero. It is clear from simulation results; FOPID tuned using N-M technique is able to minimized coupling effect more effectively as compared to 


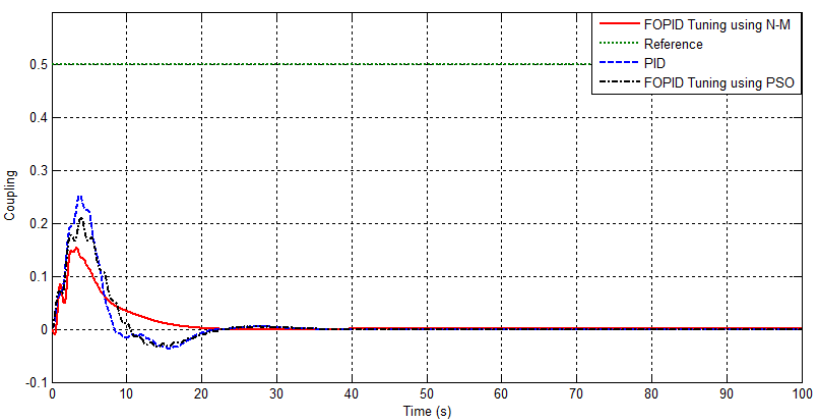

Fig. 6. Effect of cross coupling of nonlinear TRAS

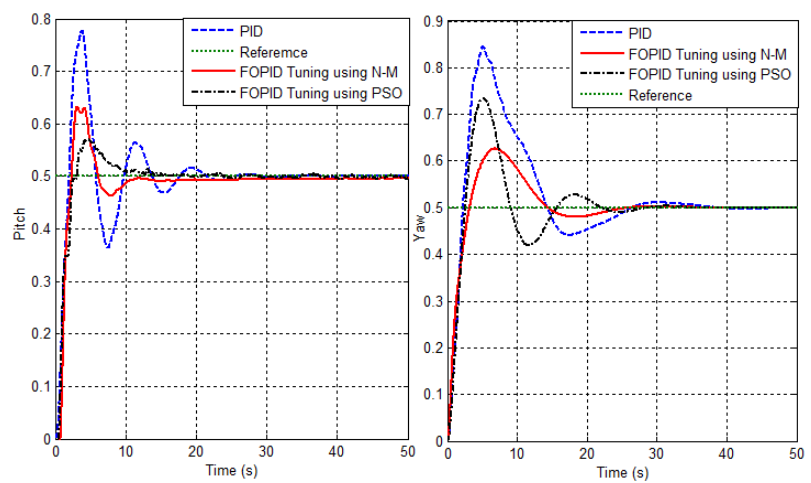

Fig. 7. Tracking performance in the presence of disturbance

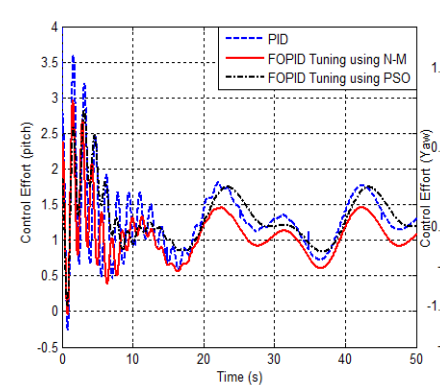

(a)

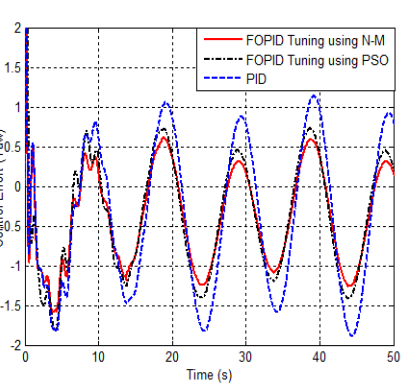

(b)
Fig. 8. Comparison of control effort of PID and FOPID controller: (a) for pitch (b) for yaw

\section{PSO and PID controller.}

Fig. 7 shows the tracking performance of TRAS system in the presence of disturbances at the control input channels of the system to demonstrate the robustness of the proposed scheme. The disturbance signals, $0.5 \sin (\psi)$ and $0.5 \sin (\phi)$, are applied at control input channels of pitch and yaw. TRAS output shows that the proposed scheme has the capability to maintain steady state in less time even in the presence of disturbance. Finally the comparison of control effort required controlling the pitch and yaw for PID and FOPID controller (N-M \& PSO) is shown in Fig. 8. Results show that FOPID controller design proposed in this paper has required less control effort compared FOPID design using PSO and PID controller.
Table 5. Comparison of FOPID and PID

\begin{tabular}{c|c|c|c|c|c|c}
\hline \multirow{2}{*}{$\begin{array}{c}\text { Performance } \\
\text { Parameters }\end{array}$} & \multicolumn{2}{|c|}{ PID } & \multicolumn{2}{c|}{$\begin{array}{c}\text { FOPID design } \\
\text { using N-M }\end{array}$} & \multicolumn{2}{c}{$\begin{array}{c}\text { FOPID design } \\
\text { using PSO }\end{array}$} \\
\cline { 2 - 7 } & Pitch & Yaw & Pitch & Yaw & Pitch & Yaw \\
\hline Settling time(sec) & 22 & 28 & 9 & 9 & 18 & 24 \\
\hline Rise Time (sec) & 4 & 3.9 & 3 & 3 & 6 & 4 \\
\hline Over Shoot (\%) & 40 & 30 & 14 & 18 & 5 & 20 \\
\hline $\begin{array}{c}\text { Steady State Error } \\
(\%)\end{array}$ & 0.1 & 0 & 0 & 0 & 0 & 0 \\
\hline
\end{tabular}

The comparison of both PID and FOPID controller according to transient performance specification is show in Table 5.

\section{Conclusion}

This paper presents the application of fractional order PID controller for accurate control of pitch and yaw angles of two degrees of freedom nonlinear TRAS model. To capture actual representation of system dynamics, the nonlinear TRAS model is first identified in fractional order transfer function representation. Grünwald-Letnikov method of approximation is used for model identification. Based upon the identified model, two separate FOPID controllers are designed to control the pitch and yaw axes of TRAS. The performance of controller is evaluated based upon transient and steady state performance specifications. Nelder-Mead (N-M) optimization technique is adopted to tune the controller parameters and is compared with PSO technique. The validity of the controller is checked by applying it on the nonlinear model of TRAS. Simulation results verified the effectiveness of FOPID controller tuned using N-M technique as compared FOPID tuned using PSO and classical PID controller. The proposed design scheme found robust in the presence of disturbance. It also required less control effort compare to classical PID and other FOPID design techniques. While evaluating performance parameters, it has been clearly analyzed the proposed scheme holds faster response and provide robust stability.

\section{Acknowledgements}

The work supported by the NSF of China under grants 61273141, 61433011 and the National Basic Research Program of China under Grant 2014CB046406.

\section{References}

[1] Igor Podlubny, "Fractional-order systems and $P I^{\lambda} D^{\mu}$ controllers," IEEE. Trans. Automation Control, vol. 44, pp. 208-214, 1999.

[2] Jiangbo Zhao, Junzheng Wang and Shoukun Wang 
"Fractional order control to the electro-hydraulic system in insulator fatigue test device," Mechatronic, vol. 23, pp. 228-239, 2013.

[3] DingyuXue, Chunna Zhao and Yan Quan Chen, "Fractional order pid control of a dc-motor with elastic shaft: a case study," In Proc. ACC, 2006, pp. 14-16.

[4] Rinku Singhal, Subhransu Padhee, Gagandeep Kaur "Design of fractional order pid controller for speed control of dc motor," Int. Jour. Scientific Research Publication, vol. 2(6), 2012.

[5] Chengbin Ma and Hori Yoichi, "The application of fractional order control to backlash vibration suppression," In Proc. ACC, 2006, pp. 2901-2906.

[6] Shivaji Karad, Dr. S. Chatterji, Prasheel Suryawanshi, "Performance analysis of fractional order pid controller with the conventional pid controller for bioreactor control," Int. Jour. Scentific Engineering Research, vol. 3(6), 2012.

[7] Cristena I. Muresan, Clara Ionescu, Silviu Folea and Robin De Keyser, "Fractional order control of unstable processes: the magnetic levitation study case," Jour. Nonlinear Dynamics, vol. 80, pp. 17611772, 2015.

[8] Magdy A.S. Aboelela' Mohamed F. Ahmed and Hassen T. Dorrah, "Design of aerospace control systems using fractional pid controller," Jour. Advance Research, vol. 3, pp. 225-232, 2012.

[9] Sunil Kumar Mishra and S Purwar, "To design optimally tuned fopid controller for twin rotor mimo system," In proc. SCES, pp. 28-30, 2014.

[10] Prasad D.G, Manoharan, P.S.; Ramalakshmi, A.P.S., "PID control scheme for twin rotor MIMO system using a real valued genetic algorithm with a predetermined search range," Int. Conf. Power, Energy and Control (ICPEC), pp. 443-448, 6-8 Feb, 2013.

[11] W.K Liu, J.H Fan and J.G Juang, "Application of system performance-index based genetic algorithm to pid controller," In Proc. AIA, 2004.

[12] Jih-Gau Juang, Kai-Ti Tu, Wen-Kai Liu, "Hybrid intelligent PID control for MIMO system," In proc. ICONIP, Springer-Verlag Berlin Heidelberg, 2006.

[13] Ji GauJuang, Ming Te Huang, Wen Kai Liu, "PID control using researched genetic algorithms for mimo system," IEEE Tran. System Man. Cyber, vol. 38(5), pp. 716-72, 2008.

[14] Abdul Qayyum Khan and Naeem Iqbal, "Modeling and design of an optimal regulator for three degree of freedom helicopter/twin rotor control system," In proc. EST, 2004.

[15] B. Pratap, A. Agrawal and S. Purwar, "Optimal control of twin rotor mimo system using output feedback," In proc $2^{\text {nd }}$ Int. Conf. Power. Control and Embedded System, 2012.

[16] Petko H. Petkov, Nicolai D. Christov and Mihail M. Konstantinov, "Robust real-time control of a two- rotor aerodynamic system," In Proc. of the 17th World Congress The International Federation of Automatic Control, Seoul, Korea, July 6-11, 2008.

[17] Peter Torvik and R.L.Bagley, "On the appearance of the fractional derivative in the behavior of real materials," Trans. ASME, vol. 51, pp. 294-298, 1984.

[18] Tom T Hartly, Carl F Lorenzob, "Fractional-order system identification based on continuous orderdistributions," Jour. Signal Processing, vol. 83, pp. 2287-2300, 2003.

[19] Islam S. M. Khalil, A. Teoman Naskali, Asif Sabanovic "On fraction order modeling and control of dynamical systems, In proc. IFAC, 2009.

[20] Lubomir Dorcak, Emmanuel A. Gonzalez, Jan Terpak, Juraj Valsa, Ladislav Pivka, "Identification of fractional- order dynamical systems based on nonlinear function optimization," Int. Jour. Pure Applied Mathematics, vol. 89, No. 2, pp. 225-250, 2013.

[21] Shantanu Das, Functional fractional calculus for system identification and controls," Springer-Verlag, Berlin Heidelberg, New York, 2008.

[22] Duarte Valerio and Jose Sa Da Costa, "A method for identifying digital models and its application to noninteger order control," In Proc. $6^{\text {th }}$ Portuguese Conference on Automatic Control IFAC, pp. 115-120, 2004.

[23] Jocelyn Sabatier, Mohamed Aoun, Alain Oustaloup, Gilles Grégoire, Franck Ragot, Patrick Roy, "Fractional system identification for lead acid battery state of charge estimation," Journal. Signal Processing, vol. 86, pp. 2645-2657, 2006.

[24] R. Lewandowski and B. Chorazy czewski, "Identification of the parameters of the kelvin-voigt and the maxwell fractional models used to modeling of viscoelastic dampers, ” Jour. Computer and Structure, vol. 88, No. 1-2, pp. 1-17, 2010.

[25] O Cois, Patrick Lanusse, Pierre Melchior and F Dancla, A. Oustaloup, "Fractional systems toolbox for matlab: applications in system identification and crone CSD, In proc. $41^{\text {st }}$ IEEE Conf. on Decision and Control, IEEE, pp. 1-6, 2002.

[26] I. Petras, D. Sierociuk and I. Podlubny, "Identification of parameters of a half-order system," IEEE Trans. Signal Processing, vol. 60, No. 10, pp. 55615566, 2012.

[27] Tomas McKelvey, Frequency domain identification methods, circuits, Systems and Signal Processing, vol. 21, No. 1, pp. 39-55, 2002.

[28] A. Dzielinski, G. Sarwas, D. Sierociuk, T. Skovranek and I. Petras, "frequency response based identification of fractional order dynamical systems," In Proc. 12 $2^{\text {th }}$ Int. Carpathian. Control Conference, 2011.

[29] P. Igor, "Fractional-order systems and $P I^{\lambda} D^{\mu}$ controllers," IEEE. Trans. Automation Control, vol. 44(1), pp. 208-14, 1999. 
[30] Hong sheng Li, Ying Luo and Yang Quang Chen, “A fractional order proportional and derivative (FOPD) motion controller: tuning rule and experiments," IEEE Transac. Control System Technology, vol. 18(2), pp. 516-520, 2010.

[31] B. M. Vinagre, I. Podlubny, A. Hernandez, V. Feliu, "Some approximations of fractional order operators used in control theory and applications, Jour. Fractional Calculus Application Analytical, vol. 3, pp. 945-950, 2004.

[32] A. Tepljakov, E. Petlenkov and J. Belikov, "FOMCON: Fractional-order modeling and control toolbox for MATLAB," In proc. $18^{\text {th }}$ Int. Conf Mixed Design Integrated Circuits and Systems, Gliwice, Poland, pp. 684-689, 2011.

[33] Duarte Valerio, "Tuning of fractional PID controllers with ziegler-nichols type rules," Jour. Signal Processing, pp. 2771-2784, 2006.

[34] J.A. Nelder, R. Mead, A simplex method for function minimization, Comp. Jour, vol. 7, pp. 308-313, 1965.

[35] Changtong Luo and Bo. Yu, "Low dimensional simplex evolution: a hybrid heuristic for global optimization" In $8^{\text {th }}$ International Conference on Software Engineering, Artificial Intelligence, Networking, and Parallel / Distributed Computing, vol. 2, pp. 470-474, 2007.

[36] Alberto Moraglio, Colin G. Johnson, "Geometric Generalization of the Nelder-Mead Algorithm," Springer-Verlag, 2010.

[37] Norlela Ishak, MazidahTajjudin, Hashimah Ismail, MohdHezriFazalulRahiman, Yahaya Md Sam, Ramli Adnan, "PID studies on position tracking control of an electro-hydraulic actuator," Int. Jour. Computer Sciences and Engineering, vol. 2(5), pp. 120-126, 2012.

[38] Dian Palup Rini, Siti Mariyam Shamsuddin, Sitiphiyati, "Particle Swarm Optimization: Technique, System and Challenges," Int. Jour. Computer Application, vol 14(1), pp. 19-27, 2011.

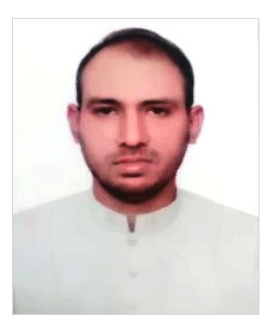

Salman Ijaz He has done his Master in Control Sciences and Engineering in 2014. He then joined COMSAT Institute of Technology Lahore as Lecturer. Currently he is enrolled in PHD in Beihang University Beijing China. His research interests are fractional order control and robust control

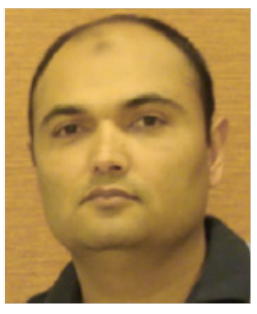

Mirza Tariq Hamayun He graduated from Eastern Mediterranean University TRNC Turkey with a B.Sc. (Hons) and M.Sc. (Hons) in Electrical Engineering in 1999 and 2001, supported by EMU TRNC Turkey. In 2001 he joined the Daimler Chrysler AG Research centre Frankfurt Germany, where he was involved in automation and measurement setup of high speed power motors. In 2004 he was employed by Comsats Institute of Information Technology Lahore as a Lecturer and promoted to Assistant Professor in 2006. In 2008 he moved to Leicester University as a Ph.D student supported by an Infrastructure planning of CIIT scholarship. He was awarded a Ph.D. in 2013, funded by a Faculty Development Program of Higher Education Commission. He is a author of over 25 refereed papers including one monograph in the area of integral sliding mode FTC.

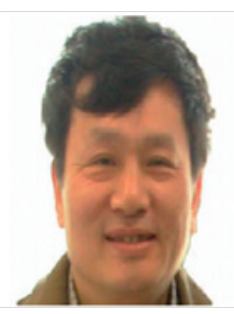

Lin Yan Lin Yan received his MS and $\mathrm{PhD}$ degrees from the Beijing University of Aeronautics and Astronautics (BUAA), Beijing, China, in 1988 and 1999, respectively. He is currently a Professor in the school of Automation, BUAA. His research interests include robust control and adaptive control..

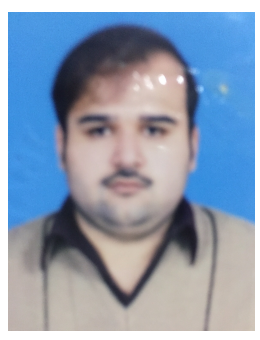

Muhammad Faisal Mumtaz $\mathrm{He}$ is $\mathrm{Ms}$ Scholar in Institute of Southern Punjab. His research interests are fractional order systems and control. 